\title{
The Minimum of a Certain Linear Form ${ }^{1}$
}

\author{
Karl Goldberg
}

(March 23, 1959)

\begin{abstract}
The positive minimum of the integral linear form $L\left(x_{1}, \ldots, x_{n}\right)=a_{1} x_{1}+\ldots+a_{n} x_{n}$ is found subject to the conditions $a_{i}>0$ and $L\left(x_{1}, \ldots, x_{n}\right) \geq 2 a_{i} x_{i}$ for $i=1,2, \ldots, n$.
\end{abstract}

Let $a_{1} \leq a_{2} \leq \ldots \leq a_{n}$ be $n \geq 3$ positive integers. We seek the positive minimum $M$ of the linear form

$$
L\left(x_{1}, x_{2}, \ldots, x_{n}\right)=a_{1} x_{1}+a_{2} x_{2}+\ldots+a_{n} x_{n}
$$

over all non-negative integers $x_{1}, x_{2}, \ldots, x_{n}$ such that

$$
L\left(x_{1}, x_{2}, \ldots ., x_{n}\right) \geq 2 a_{i} x_{i}
$$

for all $i=1,2, \ldots, n$.

Let $\left[a_{1}, a_{2}\right]$ denote the least common multiple of $a_{1}$ and $a_{2}$.

For each $i=3,4, \ldots, n$, define $r_{i}$ in the following way: If either $a_{1}$ or $a_{2}$ divides $a_{i}$, or if $a_{i}=a_{j}$ for some $j \neq i$, set $r_{i}=0$. Otherwise, let $r_{i}$ be the minimum of the least non-negative residues modulo $a_{1}$ of

$$
a_{2}-a_{i}, 2 a_{2}-a_{i}, \ldots,\left[\left(a_{i}-1\right) / a_{2}\right] a_{2}-a_{i} .
$$

We shall prove

Theorem: $M$ is the minimum of $2\left[a_{1}, a_{2}\right], 2 a_{3}+r_{3}$, $2 a_{4}+r_{4}, \ldots, 2 a_{n}+r_{n}$.

As a eonsequence we have the inequality

$$
2 a_{3}+a_{1}-1 \geq M \geq 2 a_{2} .
$$

Also, if $L\left(x_{1}, x_{2}, \ldots, x_{n}\right)=M$, then at most three of the $x_{k}$ are positive. At least two must be positive. If exactly two are positive, then either $x_{1}=\left[a_{1}, a_{2}\right] / a_{1}$ and $x_{2}=\left[a_{1}, a_{2}\right] / a_{2}$, or $x_{1}=a_{i} / a_{1}$ and $x_{i}=1$, or $x_{2}=a_{i} / a_{2}$ and $x_{i}=1$, or $x_{i}=x_{j}=1$ for some $j>i \geq 3$. If three of the $x_{k}$ are positive, then both $x_{1}$ and $x_{2}$ are positive; the other positive $x_{i}$ equals 1 and we have $x_{1}=$ $-\left[\left(a_{2} x_{2}-a_{i}\right) / a_{1}\right]$ for that $i$. Under any conditions $M$ is achieved only with $x_{i} \leq 1$ for all $i \geq 3$. We shall prove all this.

M. Newman ${ }^{2}$ refers to our theorem in the case $n=3$. We shall treat this case first.

We have $a_{1} \leq a_{2} \leq a_{3}$, and we want to find the

1 The preparation of this paper was supported in part by the Office of Naval Research.

${ }_{2}$ M. Newman, Construction and application of a class of modular functions, II, Proc. London Math. Soc. 9, 373 (1959). positive minimum $M$ of the linear form

$$
L\left(x_{1}, x_{2}, x_{3}\right)=a_{1} x_{1}+a_{2} x_{2}+a_{3} x_{3}
$$

over all non-negative integers $x_{1}, x_{2}, x_{3}$ satisf ying

$$
L\left(x_{1}, x_{2}, x_{3}\right) \geq 2 a_{i} x_{i} \quad i=1,2,3 .
$$

Let $x_{1}^{\prime}=-\left[\left(a_{2}-a_{3}\right) / a_{1}\right]$. Because $a_{2} \leq a_{3}, \quad x_{1}^{\prime}$ is non-negative. It satisfies

$$
a_{1}-1 \geq a_{2}-a_{3}+a_{1} x_{1}^{\prime} \geq 0 .
$$

Because $a_{1} \leq a_{2}$, this implies

$$
a_{3} \geq a_{3}-\left(a_{2}-a_{1}\right)-1 \geq a_{1} x_{1}^{\prime} .
$$

Now consider $L\left(x_{1}^{\prime}, 1,1\right)=a_{1} x_{1}^{\prime}+a_{2}+a_{3}$. From (3) we have

$$
2 a_{3}+a_{1}-1 \geq L\left(x_{1}^{\prime}, 1,1\right) \geq 2 a_{3} .
$$

We know that $2 a_{3} \geq 2 a_{2}$, so that $L\left(x_{1}^{\prime}, 1,1\right) \geq 2 a_{3} \geq 2 a_{2}$. Finally, (4) yields $\bar{L}\left(x_{1}^{\prime}, 1,1\right) \geq 2 a_{3} \geq 2 a_{1} x_{1}^{\prime}$.

This proves that $x_{1}=x_{1}^{\prime}, x_{2}=x_{3}=1$ satisfies (2). It follows that the left-hand inequality in (5) holds for $M$ :

$$
2 a_{3}+a_{1}-1 \geq M .
$$

From this point we assume that $x_{1}, x_{2}, x_{3}$ satisfy (2) and

$$
L\left(x_{1}, x_{2}, x_{3}\right)=M .
$$

Since $L\left(x_{1}, x_{2}, x_{3}\right) \geq 2 a_{3} x_{3}$, we have from (6) and $a_{3} \geq a_{1}$ that $x_{3}=0$ or $x_{3}=1$.

If $x_{3}=0$, than (2) implies $a_{1} x_{1}=a_{2} x_{2}$. Under this condition the minimum value of $L\left(x_{1}, x_{2}, x_{3}\right)$ is $2\left[a_{1}, a_{2}\right]$, occurring for $x_{1}=\left[a_{1}, a_{2}\right] / a_{1}$ and $x_{2}=\left[a_{1}, a_{2}\right] / a_{2}$.

From now on $x_{3}=1$. From $M=a_{1} x_{1}+a_{2} x_{2}+a_{3}$ and (2), we have $M \geq 2 a_{3}$. From (6) we have

$$
2 a_{3}+a_{1}-1 \geq 2 a_{3}+a_{1} x_{1}+\left(a_{2} x_{2}-a_{3}\right),
$$

from which it follows that $x_{1}>0$ implies $a_{3}-1 \geq a_{2} x_{2}$. If $x_{1}=0$, then (2) implies $a_{2} x_{2}=a_{3} x_{3}$, so that $M=2\left[a_{2}, a_{3}\right]$. 
But $2\left[a_{2}, a_{3}\right]>2 a_{3}+a_{1}-1$ unless $a_{2}$ divides $a_{3}$. Thus $x_{1}=0$ is possible only if $a_{2}$ divides $a_{3}$, in which case $x_{2}=a_{3} / a_{2}$ and $M=2 a_{3}$. Similariy $x_{2}=0$ is possible only if $a_{1}$ divides $a_{3}$, in which case $x_{1}=a_{3} / a_{1}$ and $M=2 a_{3}$. Since $a_{3}$ divisible by either $a_{1}$ or $a_{2}$ leads to $M=2 a_{3}$ which is the best possible result with $x_{3}=1$, we may now assume that neither $a_{1}$ nor $a_{2}$ divides $a_{3}$ and that $x_{1} x_{2}>0$.

With $x_{1}>0$ we must have $a_{3}-1 \geq a_{2} x_{2}$. Fix $x_{2}$. We shall find that permissible value of $x_{1}$ which minimizes $L\left(x_{1}, x_{2}, x_{3}\right)=a_{1} x_{1}+a_{2} x_{2}+a_{3}$. Clearly this is the least positive value of $x_{1}$ satisfying (2). We have

$$
L\left(x_{1}, x_{2}, x_{3}\right)=a_{1} x_{1}+1+\left(a_{3}-1-a_{2} x_{2}\right)+2 a_{2} x_{2}>2 a_{2} x_{2}
$$

for any value of $x_{1}$. The other inequalities require

$$
\frac{a_{3}+a_{2} x_{2}}{a_{1}} \geq x_{1} \geq \frac{a_{3}-a_{2} x_{2}}{a_{1}} .
$$

Since $2 a_{2} x_{2} \geq 2 a_{2}>a_{1}$, there are values of $x_{1}$ satisfying these inequalities. The least such $x_{1}$ is the least integer greater than or equal to $\left(a_{3}-a_{2} x_{2}\right) / a_{1}$. This last quantity is positive, so this value of $x_{1}$ is positive. It can be written

$$
x_{1}^{\prime \prime}=-\left[\frac{a_{2} x_{2}-a_{3}}{a_{1}}\right] .
$$

Let $r_{3}\left(x_{2}\right)$ be the least non-negative residue modulo $a_{1}$ of $a_{2} x_{2}-a_{3}$. Then $r_{3}\left(x_{2}\right)=a_{2} x_{2}-a_{3}+a_{1} x_{1}^{\prime \prime}$. It follows that

$$
L\left(x_{1}^{\prime \prime}, x_{2}, 1\right)=2 a_{3}+r_{3}\left(x_{2}\right) .
$$

We want the least of these values for $x_{2}$ lying between 1 and $\left[\left(a_{3}-1\right) / a_{2}\right]$. Under our assumptions on the divisibility of $a_{3}$, this is just $2 a_{3}+r_{3}$ with $r_{3}$ as defined in the theorem. This proves the theorem for $n=3$.

Now assume $n>3$. We have $a_{1} \leq a_{2} \leq \ldots \leq a_{n}$, and we want to find the positive minimum $M$ of the linear form $L\left(x_{1}, x_{2}, \ldots, x_{n}\right)$ over all non-negative integers $x_{1}, x_{2}, \ldots, x_{n}$ satisfying (1).

If $x_{1}, x_{2}, x_{3}$ satisfy $(2)$, then $x_{1}, x_{2}, x_{3}, 0, \ldots, 0$ satisfy (1). Therefore our new $M$ satisfies (6). Let

Then

$$
L\left(x_{1}, x_{2}, \ldots, x_{n}\right)=M .
$$

$2 a_{3}+a_{1}-1 \geq a_{1} x_{1}+a_{2} x_{2}+a_{3} x_{3}+\ldots+a_{n} x_{n}$

$$
\geq a_{1}\left(x_{1}+x_{2}\right)+a_{3}\left(x_{3}+\ldots .+x_{n}\right) .
$$

It follows that

$$
x_{3}+x_{4}+\ldots+x_{n} \leq 2
$$

and that $x_{3}+x_{4}+\ldots+x_{n}=2$ requires $x_{1}+x_{2}=0$. On the other hand,

$$
\begin{gathered}
3 a_{i}>2 a_{3}+a_{1}-1 \geq L\left(x_{1}, x_{2}, . . ., x_{n}\right) \geq 2 a_{i} x_{i}, \\
i=3,4, \ldots, \quad, \quad,
\end{gathered}
$$

implies

$$
x_{i} \leq 1, \quad i=3,4, \ldots, n \text {. }
$$

Assume $x_{3}+x_{4}+\ldots+x_{n}=2$. Then $x_{i}=x_{j}=1$ for some $i, j \geq 3$ and all other $x_{k}=0$. Then (1) implies $a_{i}=a_{j}$ and $M=2 a_{i}$. Again this is the best possible result with $x_{i}=1$.

If $x_{3}+x_{4}+\ldots+x_{n}=0$, then (1) implies $a_{1} x_{1}$ $=a_{2} x_{2} . \quad$ As before, this implies $M=2\left[a_{1}, a_{2}\right]$.

If $x_{3}+x_{4}+\ldots+x_{n}=1$, then $x_{i}=1$ for some $i>3$ and all other $x_{k}=0$ for $k \geq 3$. The problem then reverts to the case $n=3$ with $a_{i}$ replacing $a_{3}$. Our previous arguments complete the proof of the theorem, and the statements in the subsequent paragraph.

Washington, D.C.

(Paper 64B1-20) 